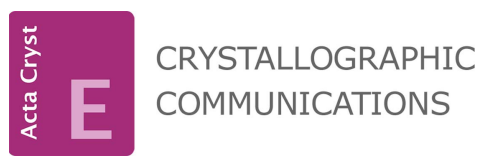

ISSN 2056-9890

Received 24 January 2015

Accepted 30 January 2015

Edited by J. Simpson, University of Otago, New Zealand

Keywords: crystal structure; imadazolidine; (2hydroxynaphthalen-1-yl)methyl; hydrogen bonding

CCDC reference: 1046536

Supporting information: this article has supporting information at journals.iucr.org/e

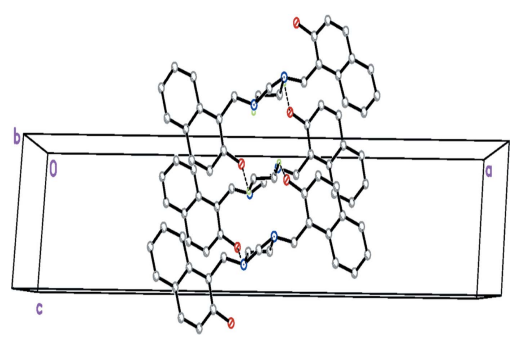

OPEN $\odot$ ACCESS

\section{Crystal structure of 1,1'-[imidazolidine-1,3- diylbis(methylene)]bis(naphthalen-2-ol)}

\author{
Augusto Rivera, ${ }^{\mathrm{a} *}$ Jicli José Rojas, ${ }^{\mathrm{a}}$ Jaime Ríos-Motta ${ }^{\mathrm{a}}$ and Michael Bolte ${ }^{\mathrm{b}}$ \\ aDepartamento de Química, Facultad de Ciencias, Universidad Nacional de Colombia, Sede Bogotá, Cra 30 No. 45-03, \\ Bogotá, Colombia, and ${ }^{\mathbf{b}}$ Institut für Anorganische Chemie, Goethe-Universität, Max-von-Laue-Strasse 7, Frankfurt/Main \\ D-60438, Germany. *Correspondence e-mail: ariverau@unal.edu.co
}

The crystal structure of the title compound, $\mathrm{C}_{25} \mathrm{H}_{24} \mathrm{~N}_{2} \mathrm{O}_{2}$, at $173 \mathrm{~K}$ has monoclinic $(C 2 / c)$ symmetry. The molecule is located on a crystallographic twofold rotation axis with only half a molecule in the asymmetric unit. The imidazolidine ring adopts a twist conformation, with a twist about the ring $\mathrm{C}-\mathrm{C}$ bond. The crystal structure shows the anticlinal disposition of the two (2hydroxynaphthalen-1-yl)methyl substituents of the imidazolidine ring. The structure displays two intramolecular $\mathrm{O}-\mathrm{H} \cdots \mathrm{N}$ hydrogen bonds, each forming an $S(6)$ ring motif.

\section{Chemical context}

We have been interested in the synthesis and characterization of a family of symmetrical $N, N^{\prime}$-disubstituted imidazolidines by the use of a Mannich-type condensation of cyclic cage aminals with phenols in a one-pot reaction. The main structural feature of the symmetrical $N, N^{\prime}$-disubstituted imidazolidines, the so-called aromatic di-Mannich bases, is to form intramolecular hydrogen bonds that reveal great structural and thermodynamic stability. These $d i$-Mannich bases which contain a phenolic or naphtholic hydroxyl group as a proton donor, as well as an ortho-aminomethyl group as a proton acceptor in the same molecule are convenient models for studying the nature of hydrogen bonding and other weak noncovalent interactions (Koll et al., 2006).<smiles>Oc1ccc2ccccc2c1CN1CCN(Cc2c(O)ccc3ccccc23)C1</smiles>

In previous studies (Rivera et al., 2006), 1,1'-[imidazolidine1,3-diylbis(methylene)]bis(naphthalen-2-ol), (I), was obtained in good yields by an one-pot Mannich-type reaction involving 1,3,6,8-tetraazatricyclo[4.4.1.1 $1^{3,8}$ dodecane (TATD) and naphthalen-2-ol in classical solvents for Mannich reactions, such as dioxane or ethanol. Intriguingly, reactions of 1,3,6,8tetraazatricyclo[4.4.1.1 $\left.{ }^{3,8}\right]$ dodecane (TATD) with naphthalen2-ol may lead to other results. It has been found (Rivera \& Quevedo, 2013) that interaction of TATD with naphthalen-2ol in solvent-free conditions by heating in an oil bath a 1:4 mixture with stirring at $423 \mathrm{~K}$ for 20 min gives $1,1^{\prime}$-methylenebis(naphthalen-2-ol) in good yields. On the other hand, the reactions of TATD with naphthalen-2-ol under solvent-free 


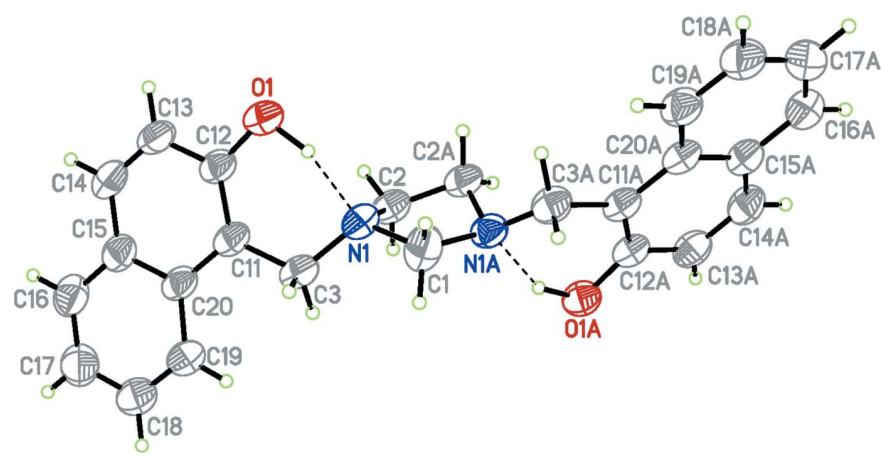

Figure 1

The molecular structure of the title compound, with displacement ellipsoids drawn at the $50 \%$ probability level. Hydrogen bonds are drawn as dashed lines. Atoms labelled with the suffix 'A' are generated using the symmetry operator $\left(-x+1, y,-z+\frac{1}{2}\right)$.

microwave-assisted conditions yields the title compound and no formation of 1,1'-methylenebis(naphthalen-2-ol) was observed. In contrast to classical Mannich reaction conditions this reaction required neither solvent nor inert atmosphere conditions.

\section{Structural commentary}

In contrast to the closely related structure (Rivera et al., $2012 a$ ), which crystallized in the monoclinic $P 2_{1} / n$ space group, the title compound crystallizes in the $C 2 / c$ space group. The molecular structure is shown in Fig. 1. The asymmetric unit contains one half molecule and the whole molecule is generated by twofold rotational symmetry (see Fig. 1). The near planarity of the fused aromatic ring system is illustrated by the very small deviation of all the atoms from the plane [largest deviation $=0.0227$ (17) $\AA$ for atom C11]. The imidazolidine ring $\left(\mathrm{C} 1 / \mathrm{N} 1 / \mathrm{C} 2 / \mathrm{C}^{\prime} / \mathrm{N} 1^{\prime}\right)$ is in a twisted conformation on $\mathrm{C} 2-$ C2', with puckering parameters $Q(2)=0.4126(17) \AA$ and $\varphi(2)$ $=126.0(2)^{\circ}($ Cremer \& Pople, 1975). The crystal structure shows the anticlinal disposition of the two (2-hydroxynaphthalen-1-yl)methyl substituents of the imidazolidine ring [pseudo-torsion angle $\mathrm{CH}_{2}-\mathrm{N} \cdots \mathrm{N}-\mathrm{CH}_{2}=-121.77(18)^{\circ}$ ]. The mean plane of the imidazolidine ring, defined by atoms $\mathrm{N} 1, \mathrm{C} 1$ and $\mathrm{N} 1^{\prime}$, makes a dihedral angle of $70.92(4)^{\circ}$ with the pendant aromatic rings $(\mathrm{C} 11-\mathrm{C} 20)$. The dihedral angle between the planes of the naphthyl rings is 60.55 (4) ${ }^{\circ}$.

As with related structures in this series, the molecular conformation is stabilized by two intramolecular $\mathrm{O}-\mathrm{H} \cdots \mathrm{N}$ hydrogen-bond interactions with $S(6)$ graph-set motifs (Bernstein et al., 1995). Due to symmetry and contrary to other structures, where hydrogen-bond distances were different, the two observed intramolecular hydrogen-bond distances were identical (Table 1).

\section{Supramolecular features}

Unlike the situation found in related structures, there is only one significant intermolecular interaction involving the $\mathrm{O}-\mathrm{H}$ group (as acceptor) and a methylene- $\mathrm{H}$ atom (as donor) to
Table 1

Hydrogen-bond geometry $\left(\AA{ }^{\circ}\right)$.

\begin{tabular}{lllll}
\hline$D-\mathrm{H} \cdots A$ & $D-\mathrm{H}$ & $\mathrm{H} \cdots A$ & $D \cdots A$ & $D-\mathrm{H} \cdots A$ \\
\hline $\mathrm{O} 1-\mathrm{H} 1 \cdots \mathrm{N} 1$ & $1.05(2)$ & $1.65(2)$ & $2.6143(19)$ & $151.0(19)$ \\
$\mathrm{C} 2-\mathrm{H} 2 A \cdots \mathrm{O} 1^{\mathrm{i}}$ & 0.99 & 2.64 & $3.257(2)$ & 121 \\
\hline
\end{tabular}

Symmetry code: (i) $x,-y+2, z-\frac{1}{2}$.

consolidate the crystal packing. These weak interactions led to the formation of parallel sets of zigzag chains extending along the $c$ axis of the crystal (Fig. 2).

\section{Database survey}

A search in the Cambridge Structural Database (Groom \& Allen, 2014) for the fragment 2,2'-[imidazolidine-1,3-diyl bis(methylene)]diphenol yielded seven hits, namely 2,2'[imidazolidine-1,3-diylbis(methylene)]bis(4-tert-butylphenol) (Rivera, Nerio \& Bolte, 2013), 2,2'-[imidazolidine-1,3-diylbis(methylene)]bis(4-chlorophenol) (Rivera et al., 2011), 2,2'[imidazolidine-1,3-diylbis(methylene)]bis[4-(2,4,4-trimethylpentan-2-yl)phenol] (Kober et al., 2012), 4,4'-difluoro-2,2' -[imidazolidine-1,3-diylbis(methylene)]diphenol (Rivera et al., 2012b) 2,2'-[imidazolidine-1,3-diylbis(methylene)]bis(6-methylphenol) (Rivera et al., 2014), 2,2'-[imidazolidine-1,3-diylbis(methylene)]diphenol (Rivera et al., 2012b) and 4,4'-dimethyl-2,2'[imidazolidine-1,3-diylbis(methylene)]diphenol (Rivera et al., 2012c). In all of these compounds, the hydroxy groups in the ortho position of the aromatic ring form an intramolecular hydrogen bond to an $\mathrm{N}$ atom of the imidazoline ring.

\section{Synthesis and crystallization}

The title compound has been synthesized in solution according to a literature procedure (Rivera et al., 2006); however, in this instance, the synthesis was carried out under microwaveassisted solvent free conditions. A mixture of $1 \mathrm{mmol}$ of 1,3,6,8-tetraazatricyclo[4.4.1.1 $\left.{ }^{3,8}\right]$ dodecane (TATD) and

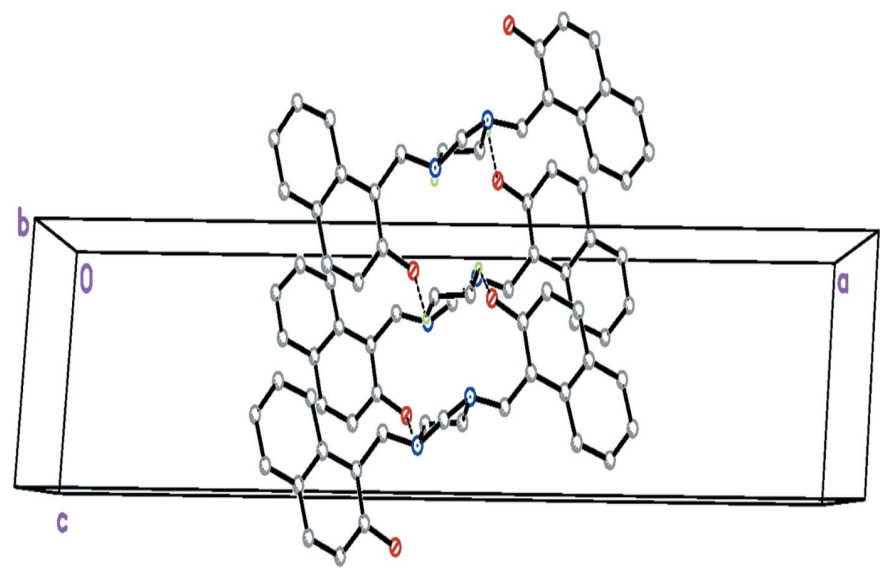

Figure 2

The crystal packing of the title compound, howing one of the zigzag chains that extend along the crystal $c$-axis direction. Hydrogen bonds are drawn as dashed lines. 
Table 2

Experimental details.

\begin{tabular}{|c|c|}
\hline \multicolumn{2}{|l|}{ Crystal data } \\
\hline Chemical formula & $\mathrm{C}_{25} \mathrm{H}_{24} \mathrm{~N}_{2} \mathrm{O}_{2}$ \\
\hline$M_{\mathrm{r}}$ & 384.46 \\
\hline Crystal system, space group & Monoclinic, $C 2 / c$ \\
\hline Temperature $(\mathrm{K})$ & 173 \\
\hline$a, b, c(\AA)$ & $34.883(5), 8.3956(9), 6.5830(8)$ \\
\hline$\beta\left(^{\circ}\right)$ & $95.650(11)$ \\
\hline$V\left(\AA^{3}\right)$ & $1918.6(4)$ \\
\hline$Z$ & 4 \\
\hline Radiation type & Mo $K \alpha$ \\
\hline$\mu\left(\mathrm{mm}^{-1}\right)$ & 0.09 \\
\hline Crystal size $(\mathrm{mm})$ & $0.19 \times 0.17 \times 0.11$ \\
\hline \multicolumn{2}{|l|}{ Data collection } \\
\hline Diffractometer & Stoe IPDS II two circle \\
\hline Absorption correction & $\begin{array}{l}\text { Multi-scan ( } X \text {-AREA; Stoe \& Cie, } \\
\text { 2001) }\end{array}$ \\
\hline$T_{\min }, T_{\max }$ & $0.972,0.989$ \\
\hline $\begin{array}{l}\text { No. of measured, independent and } \\
\text { observed }[I>2 \sigma(I)] \text { reflections }\end{array}$ & $8297,1852,1451$ \\
\hline$R_{\text {int }}$ & 0.090 \\
\hline$(\sin \theta / \lambda)_{\max }\left(\AA^{-1}\right)$ & 0.616 \\
\hline \multicolumn{2}{|l|}{ Refinement } \\
\hline$R\left[F^{2}>2 \sigma\left(F^{2}\right)\right], w R\left(F^{2}\right), S$ & $0.055,0.159,1.09$ \\
\hline No. of reflections & 1852 \\
\hline No. of parameters & 136 \\
\hline $\mathrm{H}$-atom treatment & $\begin{array}{l}\mathrm{H} \text { atoms treated by a mixture of } \\
\text { independent and constrained } \\
\text { refinement }\end{array}$ \\
\hline$\Delta \rho_{\max }, \Delta \rho_{\min }\left(\mathrm{e} \AA^{-3}\right)$ & $0.24,-0.23$ \\
\hline
\end{tabular}

Computer programs: $X$-AREA (Stoe \& Cie, 2001), SHELXS97 and XP (Sheldrick, 2008), SHELXL2014 (Sheldrick, 2015) and publCIF (Westrip, 2010).

2 mmol of naphthalen-2-ol was subjected to microwave irradiation $(200 \mathrm{~W})$ for $10 \mathrm{~min}$ at a temperature of $373 \mathrm{~K}$. The product was washed with water and then with benzene (yield 94\%, m.p. 435-436 K). Crystals suitable for X-ray diffraction were obtained from a methanol solution upon slow evaporation of the solvent at room temperature.

\section{Refinement details}

Crystal data, data collection and structure refinement details are summarized in Table 2. All $\mathrm{H}$ atoms were located in the difference electron-density map. The hydroxy $\mathrm{H}$ atom was refined freely, while $\mathrm{C}$-bound $\mathrm{H}$ atoms were fixed geometrically $(\mathrm{C}-\mathrm{H}=0.95$ or $0.99 \AA)$ and refined using a riding model, with $U_{\text {iso }}(\mathrm{H})$ values set at $1.2 U_{\text {eq }}$ of the parent atom.

\section{Acknowledgements}

We acknowledge the financial support provided to us by the Dirección de Investigación, Sede Bogotá (DIB) at the Universidad Nacional de Colombia through the research project No. 19151 (Code QUIPU 201010020518). JJR thanks COLCIENCIAS for a fellowship.

\section{References}

Bernstein, J., Davis, R. E., Shimoni, L. \& Chang, N.-L. (1995). Angew. Chem. Int. Ed. Engl. 34, 1555-1573.

Cremer, D. \& Pople, J. A. (1975). J. Am. Chem. Soc. 97, 1354-1358.

Groom, C. R. \& Allen, F. H. (2014). Angew. Chem. Int. Ed. 53, 662 671.

Kober, E., Nerkowski, T., Janas, Z. \& Jerzykiewicz, L. B. (2012). Dalton Trans. 41, 5188-5192.

Koll, A., Karpfen, A. \& Wolschann, P. (2006). J. Mol. Struct. 790, 5564.

Rivera, A., Nerio, L. S. \& Bolte, M. (2013). Acta Cryst. E69, o1166.

Rivera, A., Nerio, L. S. \& Bolte, M. (2014). Acta Cryst. E70, o243.

Rivera, A., Nerio, L. S., Ríos-Motta, J., Fejfarová, K. \& Dušek, M. (2012a). Acta Cryst. E68, o170-o171.

Rivera, A., Nerio, L. S., Ríos-Motta, J., Kučeráková, M. \& Dušek, M. (2012b). Acta Cryst. E68, o3043-03044.

Rivera, A., Nerio, L. S., Ríos-Motta, J., Kučeraková, M. \& Dušek, M. (2012c). Acta Cryst. E68, o3172.

Rivera, A. \& Quevedo, R. (2013). Tetrahedron Lett. 54, 1416-1420.

Rivera, A., Ríos-Motta, J. \& Navarro, M. A. (2006). Heterocycles, 68 , 531-537.

Rivera, A., Sadat-Bernal, J., Ríos-Motta, J., Pojarová, M. \& Dušek, M. (2011). Acta Cryst. E67, o2581.

Sheldrick, G. M. (2008). Acta Cryst. A64, 112-122.

Sheldrick, G. M. (2015). Acta Cryst. C71, 3-8.

Stoe \& Cie (2001). X-AREA. Stoe \& Cie, Darmstadt, Germany.

Westrip, S. P. (2010). J. Appl. Cryst. 43, 920-925. 


\section{supporting information}

Acta Cryst. (2015). E71, 258-260 [doi:10.1107/S2056989015002078]

\section{Crystal structure of 1,1'-[imidazolidine-1,3-diylbis(methylene)]bis- (naphthalen-2-ol)}

\section{Augusto Rivera, Jicli José Rojas, Jaime Ríos-Motta and Michael Bolte}

\section{Computing details}

Data collection: $X$-AREA (Stoe \& Cie, 2001); cell refinement: $X$-AREA (Stoe \& Cie, 2001); data reduction: $X$-AREA (Stoe \& Cie, 2001); program(s) used to solve structure: SHELXS97 (Sheldrick, 2008); program(s) used to refine structure: SHELXL2014 (Sheldrick, 2015); molecular graphics: XP (Sheldrick, 2008); software used to prepare material for publication: SHELXL2014 (Sheldrick, 2015) and publCIF (Westrip, 2010).

1-(\{3-[(2-Hydroxynaphthalen-1-yl)methyl]imidazolidin-1-yl\}methyl)naphthalen-2-ol

Crystal data

$\mathrm{C}_{25} \mathrm{H}_{24} \mathrm{~N}_{2} \mathrm{O}_{2}$

$M_{r}=384.46$

Monoclinic, $C 2 / c$

$a=34.883(5) \AA$

$b=8.3956$ (9) $\AA$

$c=6.5830(8) \AA$

$\beta=95.650(11)^{\circ}$

$V=1918.6(4) \AA^{3}$

$Z=4$

Data collection

Stoe IPDS II two-circle diffractometer

$\omega$ scans

Absorption correction: multi-scan

$X$-AREA (Stoe \& Cie, 2001)

$T_{\min }=0.972, T_{\max }=0.989$

8297 measured reflections

\section{Refinement}

Refinement on $F^{2}$

Least-squares matrix: full

$R\left[F^{2}>2 \sigma\left(F^{2}\right)\right]=0.055$

$w R\left(F^{2}\right)=0.159$

$S=1.09$

1852 reflections

136 parameters

0 restraints
$F(000)=816$

$D_{\mathrm{x}}=1.331 \mathrm{Mg} \mathrm{m}^{-3}$

Mo $K \alpha$ radiation, $\lambda=0.71073 \AA$

Cell parameters from 8026 reflections

$\theta=2.4-26.2^{\circ}$

$\mu=0.09 \mathrm{~mm}^{-1}$

$T=173 \mathrm{~K}$

Block, colourless

$0.19 \times 0.17 \times 0.11 \mathrm{~mm}$

1852 independent reflections

1451 reflections with $I>2 \sigma(I)$

$R_{\text {int }}=0.090$

$\theta_{\max }=26.0^{\circ}, \theta_{\min }=2.5^{\circ}$

$h=-42 \rightarrow 34$

$k=-10 \rightarrow 10$

$l=-8 \rightarrow 8$

Hydrogen site location: mixed

$\mathrm{H}$ atoms treated by a mixture of independent and constrained refinement

$w=1 /\left[\sigma^{2}\left(F_{0}^{2}\right)+(0.085 P)^{2}+0.4089 P\right]$ where $P=\left(F_{\mathrm{o}}^{2}+2 F_{\mathrm{c}}^{2}\right) / 3$

$(\Delta / \sigma)_{\max }<0.001$

$\Delta \rho_{\max }=0.24 \mathrm{e} \AA^{-3}$

$\Delta \rho_{\min }=-0.23 \mathrm{e} \AA^{-3}$ 


\section{Special details}

Geometry. All e.s.d.'s (except the e.s.d. in the dihedral angle between two 1.s. planes) are estimated using the full covariance matrix. The cell e.s.d.'s are taken into account individually in the estimation of e.s.d.'s in distances, angles and torsion angles; correlations between e.s.d.'s in cell parameters are only used when they are defined by crystal symmetry. An approximate (isotropic) treatment of cell e.s.d.'s is used for estimating e.s.d.'s involving l.s. planes.

Fractional atomic coordinates and isotropic or equivalent isotropic displacement parameters $\left(\hat{A}^{2}\right)$

\begin{tabular}{llllll}
\hline & $x$ & $y$ & $z$ & $U_{\text {iso }}{ }^{*} / U_{\text {eq }}$ & Occ. $(<1)$ \\
\hline O1 & $0.45368(4)$ & $0.77294(16)$ & $0.70176(19)$ & $0.0507(4)$ & \\
H1 & $0.4677(6)$ & $0.723(3)$ & $0.583(4)$ & $0.061(6)^{*}$ & \\
N1 & $0.47179(4)$ & $0.69601(18)$ & $0.3384(2)$ & $0.0455(4)$ & \\
C1 & 0.5000 & $0.5948(4)$ & 0.2500 & $0.0627(8)$ & \\
H1A & 0.5132 & 0.5258 & 0.3570 & $0.075^{*}$ & 0.5 \\
H1B & 0.4868 & 0.5258 & 0.1430 & $0.075^{*}$ & 0.5 \\
C2 & $0.47856(5)$ & $0.8543(2)$ & $0.2548(2)$ & $0.0488(5)$ & \\
H2A & 0.4707 & 0.9396 & 0.3459 & $0.059^{*}$ & \\
H2B & 0.4646 & 0.8678 & 0.1175 & $0.059^{*}$ & \\
C3 & $0.43203(5)$ & $0.6374(2)$ & $0.2997(3)$ & $0.0491(5)$ & \\
H3A & 0.4319 & 0.5212 & 0.3249 & $0.059^{*}$ & \\
H3B & 0.4231 & 0.6549 & 0.1540 & $0.059^{*}$ & \\
C11 & $0.40403(5)$ & $0.7157(2)$ & $0.4287(2)$ & $0.0437(4)$ & \\
C12 & $0.41619(5)$ & $0.7796(2)$ & $0.6188(2)$ & $0.0446(4)$ & \\
C13 & $0.38994(6)$ & $0.8541(2)$ & $0.7379(3)$ & $0.0511(5)$ & \\
H13 & 0.3990 & 0.8993 & 0.8660 & $0.061^{*}$ & \\
C14 & $0.35197(6)$ & $0.8623(2)$ & $0.6724(3)$ & $0.0541(5)$ & \\
H14 & 0.3348 & 0.9149 & 0.7536 & $0.065^{*}$ & \\
C15 & $0.33758(5)$ & $0.7931(2)$ & $0.4833(3)$ & $0.0499(5)$ & \\
C16 & $0.29795(6)$ & $0.7964(3)$ & $0.4150(3)$ & $0.0611(6)$ & \\
H16 & 0.2805 & 0.8489 & 0.4948 & $0.073^{*}$ & \\
C17 & $0.28426(6)$ & $0.7252(3)$ & $0.2355(3)$ & $0.0682(6)$ & \\
H17 & 0.2575 & 0.7286 & 0.1910 & $0.082^{*}$ & \\
C18 & $0.30975(6)$ & $0.6476(3)$ & $0.1178(3)$ & $0.0662(6)$ & \\
H18 & 0.3001 & 0.5965 & -0.0055 & $0.079^{*}$ & \\
C19 & $0.34828(6)$ & $0.6438(3)$ & $0.1770(3)$ & $0.0545(5)$ & \\
H19 & 0.3650 & 0.5908 & 0.0938 & $0.065^{*}$ & \\
C20 & $0.36387(5)$ & $0.7181(2)$ & $0.3618(2)$ & & \\
& & & & \\
\end{tabular}

Atomic displacement parameters $\left(\AA^{2}\right)$

\begin{tabular}{lllllll}
\hline & $U^{11}$ & $U^{22}$ & $U^{33}$ & $U^{12}$ & $U^{13}$ & $U^{23}$ \\
\hline O1 & $0.0588(8)$ & $0.0562(8)$ & $0.0367(6)$ & $-0.0007(6)$ & $0.0024(5)$ & $-0.0039(5)$ \\
N1 & $0.0551(9)$ & $0.0469(8)$ & $0.0350(7)$ & $0.0057(6)$ & $0.0072(6)$ & $-0.0001(6)$ \\
C1 & $0.0688(18)$ & $0.0559(17)$ & $0.0670(17)$ & 0.000 & $0.0256(14)$ & 0.000 \\
C2 & $0.0617(11)$ & $0.0505(11)$ & $0.0342(8)$ & $0.0027(8)$ & $0.0047(7)$ & $0.0023(7)$ \\
C3 & $0.0615(12)$ & $0.0496(10)$ & $0.0364(8)$ & $-0.0001(8)$ & $0.0061(7)$ & $-0.0058(7)$ \\
C11 & $0.0590(11)$ & $0.0412(9)$ & $0.0318(8)$ & $0.0009(8)$ & $0.0086(7)$ & $0.0016(6)$ \\
C12 & $0.0574(11)$ & $0.0438(10)$ & $0.0331(8)$ & $-0.0026(7)$ & $0.0067(7)$ & $0.0024(6)$
\end{tabular}


supporting information

\begin{tabular}{lllllll}
$\mathrm{C} 13$ & $0.0676(12)$ & $0.0525(11)$ & $0.0342(8)$ & $-0.0042(9)$ & $0.0098(8)$ & $-0.0063(7)$ \\
$\mathrm{C} 14$ & $0.0667(12)$ & $0.0548(11)$ & $0.0436(9)$ & $0.0030(9)$ & $0.0192(8)$ & $-0.0039(8)$ \\
$\mathrm{C} 15$ & $0.0570(11)$ & $0.0539(11)$ & $0.0399(9)$ & $-0.0012(8)$ & $0.0107(7)$ & $0.0054(7)$ \\
$\mathrm{C} 16$ & $0.0597(12)$ & $0.0746(14)$ & $0.0508(11)$ & $0.0032(10)$ & $0.0141(9)$ & $0.0079(9)$ \\
$\mathrm{C} 17$ & $0.0549(12)$ & $0.0951(18)$ & $0.0539(12)$ & $-0.0018(11)$ & $0.0020(9)$ & $0.0091(11)$ \\
$\mathrm{C} 18$ & $0.0678(14)$ & $0.0890(16)$ & $0.0409(10)$ & $-0.0065(12)$ & $0.0010(9)$ & $0.0002(10)$ \\
$\mathrm{C} 19$ & $0.0614(12)$ & $0.0646(12)$ & $0.0376(9)$ & $-0.0022(9)$ & $0.0055(8)$ & $-0.0020(8)$ \\
$\mathrm{C} 20$ & $0.0602(11)$ & $0.0455(10)$ & $0.0316(8)$ & $-0.0021(8)$ & $0.0082(7)$ & $0.0036(6)$ \\
\hline
\end{tabular}

Geometric parameters $\left(\AA,{ }^{\circ}\right)$

\begin{tabular}{|c|c|c|c|}
\hline $\mathrm{O} 1-\mathrm{C} 12$ & $1.368(2)$ & $\mathrm{C} 12-\mathrm{C} 13$ & $1.409(3)$ \\
\hline $\mathrm{O} 1-\mathrm{H} 1$ & $1.05(2)$ & $\mathrm{C} 13-\mathrm{C} 14$ & $1.354(3)$ \\
\hline $\mathrm{N} 1-\mathrm{C} 1$ & $1.464(2)$ & $\mathrm{C} 13-\mathrm{H} 13$ & 0.9500 \\
\hline $\mathrm{N} 1-\mathrm{C} 2$ & $1.467(2)$ & $\mathrm{C} 14-\mathrm{C} 15$ & $1.420(3)$ \\
\hline $\mathrm{N} 1-\mathrm{C} 3$ & $1.470(2)$ & $\mathrm{C} 14-\mathrm{H} 14$ & 0.9500 \\
\hline $\mathrm{C} 1-\mathrm{N} 1^{\mathrm{i}}$ & $1.464(2)$ & $\mathrm{C} 15-\mathrm{C} 16$ & $1.411(3)$ \\
\hline $\mathrm{C} 1-\mathrm{H} 1 \mathrm{~A}$ & 0.9900 & $\mathrm{C} 15-\mathrm{C} 20$ & $1.422(3)$ \\
\hline $\mathrm{C} 1-\mathrm{H} 1 \mathrm{~B}$ & 0.9900 & $\mathrm{C} 16-\mathrm{C} 17$ & $1.368(3)$ \\
\hline $\mathrm{C} 2-\mathrm{C}^{2}{ }^{\mathrm{i}}$ & $1.503(4)$ & $\mathrm{C} 16-\mathrm{H} 16$ & 0.9500 \\
\hline $\mathrm{C} 2-\mathrm{H} 2 \mathrm{~A}$ & 0.9900 & $\mathrm{C} 17-\mathrm{C} 18$ & $1.397(3)$ \\
\hline $\mathrm{C} 2-\mathrm{H} 2 \mathrm{~B}$ & 0.9900 & $\mathrm{C} 17-\mathrm{H} 17$ & 0.9500 \\
\hline $\mathrm{C} 3-\mathrm{C} 11$ & $1.507(2)$ & $\mathrm{C} 18-\mathrm{C} 19$ & $1.362(3)$ \\
\hline $\mathrm{C} 3-\mathrm{H} 3 \mathrm{~A}$ & 0.9900 & $\mathrm{C} 18-\mathrm{H} 18$ & 0.9500 \\
\hline $\mathrm{C} 3-\mathrm{H} 3 \mathrm{~B}$ & 0.9900 & $\mathrm{C} 19-\mathrm{C} 20$ & $1.427(2)$ \\
\hline $\mathrm{C} 11-\mathrm{C} 12$ & $1.389(2)$ & C19-H19 & 0.9500 \\
\hline $\mathrm{C} 11-\mathrm{C} 20$ & $1.427(3)$ & & \\
\hline $\mathrm{C} 12-\mathrm{O} 1-\mathrm{H} 1$ & $102.4(12)$ & $\mathrm{O} 1-\mathrm{C} 12-\mathrm{C} 13$ & $116.36(15)$ \\
\hline $\mathrm{C} 1-\mathrm{N} 1-\mathrm{C} 2$ & $103.73(15)$ & $\mathrm{C} 11-\mathrm{C} 12-\mathrm{C} 13$ & $121.01(17)$ \\
\hline $\mathrm{C} 1-\mathrm{N} 1-\mathrm{C} 3$ & $113.36(14)$ & $\mathrm{C} 14-\mathrm{C} 13-\mathrm{C} 12$ & $120.94(16)$ \\
\hline $\mathrm{C} 2-\mathrm{N} 1-\mathrm{C} 3$ & $114.98(14)$ & $\mathrm{C} 14-\mathrm{C} 13-\mathrm{H} 13$ & 119.5 \\
\hline $\mathrm{N} 1 \mathrm{i}-\mathrm{C} 1-\mathrm{N} 1$ & $109.0(2)$ & $\mathrm{C} 12-\mathrm{C} 13-\mathrm{H} 13$ & 119.5 \\
\hline $\mathrm{N} 1-\mathrm{C} 1-\mathrm{H} 1 \mathrm{~A}$ & 109.9 & $\mathrm{C} 13-\mathrm{C} 14-\mathrm{C} 15$ & $120.60(17)$ \\
\hline $\mathrm{N} 1-\mathrm{C} 1-\mathrm{H} 1 \mathrm{~A}$ & 109.9 & $\mathrm{C} 13-\mathrm{C} 14-\mathrm{H} 14$ & 119.7 \\
\hline $\mathrm{N} 1-\mathrm{C} 1-\mathrm{H} 1 \mathrm{~B}$ & 109.9 & $\mathrm{C} 15-\mathrm{C} 14-\mathrm{H} 14$ & 119.7 \\
\hline $\mathrm{N} 1-\mathrm{C} 1-\mathrm{H} 1 \mathrm{~B}$ & 109.9 & $\mathrm{C} 16-\mathrm{C} 15-\mathrm{C} 14$ & $121.46(18)$ \\
\hline $\mathrm{H} 1 \mathrm{~A}-\mathrm{C} 1-\mathrm{H} 1 \mathrm{~B}$ & 108.3 & $\mathrm{C} 16-\mathrm{C} 15-\mathrm{C} 20$ & $119.72(18)$ \\
\hline $\mathrm{N} 1-\mathrm{C} 2-\mathrm{C} 2^{\mathrm{i}}$ & $102.34(10)$ & $\mathrm{C} 14-\mathrm{C} 15-\mathrm{C} 20$ & $118.82(18)$ \\
\hline $\mathrm{N} 1-\mathrm{C} 2-\mathrm{H} 2 \mathrm{~A}$ & 111.3 & $\mathrm{C} 17-\mathrm{C} 16-\mathrm{C} 15$ & $120.9(2)$ \\
\hline $\mathrm{C} 22^{\mathrm{i}}-\mathrm{C} 2-\mathrm{H} 2 \mathrm{~A}$ & 111.3 & $\mathrm{C} 17-\mathrm{C} 16-\mathrm{H} 16$ & 119.5 \\
\hline $\mathrm{N} 1-\mathrm{C} 2-\mathrm{H} 2 \mathrm{~B}$ & 111.3 & $\mathrm{C} 15-\mathrm{C} 16-\mathrm{H} 16$ & 119.5 \\
\hline $\mathrm{C} 2{ }^{\mathrm{i}}-\mathrm{C} 2-\mathrm{H} 2 \mathrm{~B}$ & 111.3 & $\mathrm{C} 16-\mathrm{C} 17-\mathrm{C} 18$ & $119.7(2)$ \\
\hline $\mathrm{H} 2 \mathrm{~A}-\mathrm{C} 2-\mathrm{H} 2 \mathrm{~B}$ & 109.2 & $\mathrm{C} 16-\mathrm{C} 17-\mathrm{H} 17$ & 120.1 \\
\hline $\mathrm{N} 1-\mathrm{C} 3-\mathrm{C} 11$ & $114.13(14)$ & $\mathrm{C} 18-\mathrm{C} 17-\mathrm{H} 17$ & 120.1 \\
\hline $\mathrm{N} 1-\mathrm{C} 3-\mathrm{H} 3 \mathrm{~A}$ & 108.7 & $\mathrm{C} 19-\mathrm{C} 18-\mathrm{C} 17$ & $121.1(2)$ \\
\hline $\mathrm{C} 11-\mathrm{C} 3-\mathrm{H} 3 \mathrm{~A}$ & 108.7 & $\mathrm{C} 19-\mathrm{C} 18-\mathrm{H} 18$ & 119.5 \\
\hline $\mathrm{N} 1-\mathrm{C} 3-\mathrm{H} 3 \mathrm{~B}$ & 108.7 & $\mathrm{C} 17-\mathrm{C} 18-\mathrm{H} 18$ & 119.5 \\
\hline
\end{tabular}




$\begin{array}{llll}\mathrm{C} 11-\mathrm{C} 3-\mathrm{H} 3 \mathrm{~B} & 108.7 & \mathrm{C} 18-\mathrm{C} 19-\mathrm{C} 20 & 121.08(19) \\ \mathrm{H} 3 \mathrm{~A}-\mathrm{C} 3-\mathrm{H} 3 \mathrm{~B} & 107.6 & \mathrm{C} 18-\mathrm{C} 19-\mathrm{H} 19 & 119.5 \\ \mathrm{C} 12-\mathrm{C} 11-\mathrm{C} 20 & 118.43(16) & \mathrm{C} 20-\mathrm{C} 19-\mathrm{H} 19 & 119.5 \\ \mathrm{C} 12-\mathrm{C} 11-\mathrm{C} 3 & 121.27(17) & \mathrm{C} 15-\mathrm{C} 20-\mathrm{C} 11 & 117.07(16) \\ \mathrm{C} 20-\mathrm{C} 11-\mathrm{C} 3 & 120.22(15) & \mathrm{C} 15-\mathrm{C} 20-\mathrm{C} 19 & 122.50(17) \\ \mathrm{O} 1-\mathrm{C} 12-\mathrm{C} 11 & 122.62(16) & \mathrm{C} 11-\mathrm{C} 20-\mathrm{C} 19 & -1.4(3) \\ \mathrm{C} 2-\mathrm{N} 1-\mathrm{C} 1-\mathrm{N} 1 \mathrm{i} & 13.70(8) & \mathrm{C} 13-\mathrm{C} 14-\mathrm{C} 15-\mathrm{C} 20 & -178.1(2) \\ \mathrm{C} 3-\mathrm{N} 1-\mathrm{C} 1-\mathrm{N} 1 \mathrm{i} & 139.08(14) & \mathrm{C} 14-\mathrm{C} 15-\mathrm{C} 16-\mathrm{C} 17 & 1.6(3) \\ \mathrm{C} 1-\mathrm{N} 1-\mathrm{C} 2-\mathrm{C} 2 \mathrm{i} & -34.89(17) & \mathrm{C} 20-\mathrm{C} 15-\mathrm{C} 16-\mathrm{C} 17 & 0.2(4) \\ \mathrm{C} 3-\mathrm{N} 1-\mathrm{C} 2-\mathrm{C} 2 \mathrm{i} & -159.24(14) & \mathrm{C} 15-\mathrm{C} 16-\mathrm{C} 17-\mathrm{C} 18 & -1.2(4) \\ \mathrm{C} 1-\mathrm{N} 1-\mathrm{C} 3-\mathrm{C} 11 & 166.25(14) & \mathrm{C} 16-\mathrm{C} 17-\mathrm{C} 18-\mathrm{C} 19 & 0.4(3) \\ \mathrm{C} 2-\mathrm{N} 1-\mathrm{C} 3-\mathrm{C} 11 & -74.64(18) & \mathrm{C} 17-\mathrm{C} 18-\mathrm{C} 19-\mathrm{C} 20 & 179.33(16) \\ \mathrm{N} 1-\mathrm{C} 3-\mathrm{C} 11-\mathrm{C} 12 & -26.7(2) & \mathrm{C} 16-\mathrm{C} 15-\mathrm{C} 20-\mathrm{C} 11 & -1.0(3) \\ \mathrm{N} 1-\mathrm{C} 3-\mathrm{C} 11-\mathrm{C} 20 & 156.64(15) & \mathrm{C} 14-\mathrm{C} 15-\mathrm{C} 20-\mathrm{C} 11 & -2.2(3) \\ \mathrm{C} 20-\mathrm{C} 11-\mathrm{C} 12-\mathrm{O} 1 & 175.45(15) & \mathrm{C} 16-\mathrm{C} 15-\mathrm{C} 20-\mathrm{C} 19 & 177.39(17) \\ \mathrm{C} 3-\mathrm{C} 11-\mathrm{C} 12-\mathrm{O} 1 & -1.3(3) & \mathrm{C} 14-\mathrm{C} 15-\mathrm{C} 20-\mathrm{C} 19 & 3.6(3) \\ \mathrm{C} 20-\mathrm{C} 11-\mathrm{C} 12-\mathrm{C} 13 & -3.9(3) & \mathrm{C} 12-\mathrm{C} 11-\mathrm{C} 20-\mathrm{C} 15 & -179.57(16) \\ \mathrm{C} 3-\mathrm{C} 11-\mathrm{C} 12-\mathrm{C} 13 & 179.33(16) & \mathrm{C} 3-\mathrm{C} 11-\mathrm{C} 20-\mathrm{C} 15 & -174.69(17) \\ \mathrm{O} 1-\mathrm{C} 12-\mathrm{C} 13-\mathrm{C} 14 & -177.87(16) & \mathrm{C} 12-\mathrm{C} 11-\mathrm{C} 20-\mathrm{C} 19 & 2.1(3) \\ \mathrm{C} 11-\mathrm{C} 12-\mathrm{C} 13-\mathrm{C} 14 & 1.5(3) & \mathrm{C} 3-\mathrm{C} 11-\mathrm{C} 20-\mathrm{C} 19 & 1.3(3) \\ \mathrm{C} 12-\mathrm{C} 13-\mathrm{C} 14-\mathrm{C} 15 & 1.2(3) & \mathrm{C} 18-\mathrm{C} 19-\mathrm{C} 20-\mathrm{C} 15 & 179.66(18) \\ \mathrm{C} 13-\mathrm{C} 14-\mathrm{C} 15-\mathrm{C} 16 & 178.20(18) & \mathrm{C} 18-\mathrm{C} 19-\mathrm{C} 20-\mathrm{C} 11 & \\ & & & \end{array}$

Symmetry code: (i) $-x+1, y,-z+1 / 2$.

Hydrogen-bond geometry $\left(\AA,{ }^{o}\right)$

\begin{tabular}{lllll}
\hline$D-\mathrm{H} \cdots A$ & $D-\mathrm{H}$ & $\mathrm{H} \cdots A$ & $D \cdots A$ & $D-\mathrm{H} \cdots A$ \\
\hline $\mathrm{O} 1-\mathrm{H} 1 \cdots \mathrm{N} 1$ & $1.05(2)$ & $1.65(2)$ & $2.6143(19)$ & $151.0(19)$ \\
$\mathrm{C} 2-\mathrm{H} 2 A \cdots \mathrm{O} 1{ }^{\mathrm{ii}}$ & 0.99 & 2.64 & $3.257(2)$ & 121 \\
\hline
\end{tabular}

Symmetry code: (ii) $x,-y+2, z-1 / 2$. 